\title{
Amazônia no Antropoceno: o manejo como relação entre humanos e fauna silvestre
}

Amazon in the Anthropocene: management as a relationship between humans and wildlife

\section{Amazon dans l'anthropocène: la gestion en tant que relation entre les humains et la faune}

\section{Amazonia en el Antropoceno: el manejo como relación entre humanos y fauna silvestre}

\author{
Wagner de Deus Mateus ${ }^{1}$ \\ Mariana Baldoino ${ }^{1}$ \\ Elisa Ferrari Justulin Zacarias ${ }^{1}$ \\ Maria Inês Gasparetto Higuchi
}

Recebido em 05/08/2017; revisado e aprovado em 24/09/2017; aceito em 04/10/2017

DOI: http://dx.doi.org/10.20435/inter.v19i3.1667

\begin{abstract}
Resumo: O trabalho foi realizado para discutir a conservação e manejo da fauna silvestre amazônica a partir da relação entre humano e não humano como respostas a defaunação na época do Antropoceno. Na Amazônia, essas práticas transformam o modo como as populações humanas se relacionam com as espécies pirarucu, quelônios e jacarés.
\end{abstract}

Palavras-chave: Antropoceno; defaunação; conservação; manejo; Amazônia.

Abstract: The work was carried out to discuss the conservation and management of Amazonian wild fauna from the relationship between human and nonhuman as responses to defaunation in the Anthropocene era. In the Amazon these practices transform the way human populations relate to pirarucu, chelonians and alligators.

Keywords: Anthropocene; defaunation; conservation; management; Amazon.

Résumé: Le travail a été mené pour discuter de la conservation et de la gestion de la faune sauvage amazonienne à partir de la relation entre les humains et les non-humains en tant que réponses à la défaillance à l'ère de l'anthropocène. En Amazonie, ces pratiques transforment la façon dont les populations humaines se rapportent aux pirarucu, aux chéloniens et aux alligators.

Mots-clés: Anthropocène; défaunation; conservation; gestion; Amazon.

Resumen: El trabajo fue realizado para discutir la conservación y manejo de la fauna silvestre amazónica a partir de la relación entre humano y no humano como respuestas a la defaunación en la época del Antropoceno. En la Amazonia estas prácticas transforman el modo en que las poblaciones humanas se relacionan con las especies pirarucu, quelonios y caimanes.

Palabras clave: Antropoceno; defaunación; conservación; manejo; Amazonia.

\section{INTRODUÇÃO}

A região amazônica é considerada megadiversa pelo volume de plantas superiores (40.000 espécies), mamíferos (427 espécies), aves (1.300 espécies), répteis (378 espécies), anfíbios (427 espécies) e peixes de água doce ( 3.500 espécies) (WORLD WIDE FUND FOR NATURE [WWF], 2010; KRITSCH, 2009). Contudo, assim como no cenário global, vivencia a perda de espécies

\footnotetext{
${ }^{1}$ Universidade Federal do Amazonas (UFAM), Manaus, Amazonas, Brasil.

2 Instituto Nacional de Pesquisas da Amazônia (INPA), Laboratório de Psicologia e Educação Ambiental, Manaus, Amazonas, Brasil.
} 
silvestres ocasionadas pela ação humana identificada como a sexta grande defaunação do Antropoceno (DIRZO et al., 2014; CEBALLOS; EHRLICH; DIRZO, 2017).

A época do antropoceno é catalisadora da recaracterização das formas de relação entre humanos e não humanos. A defaunação em curso nessa época significa a perda da fauna silvestre e, com ela, a segurança alimentar de milhares ribeirinhos amazônicos que dependem de inúmeras espécies animais e vegetais. Nesse sentido, o desenvolvimento de modelos de manejos conservacionistas locais surge como estratégia para enfrentar a extinção da fauna e beneficiar socioeconomicamente ribeirinhos e não ribeirinhos. Dessa forma, faz-se necessário o debate acerca do Antropoceno, defaunação, conservação e manejo da fauna silvestre e como esses processos atuam na reformulação das relações entre ribeirinhos e espécies silvestres amazônicas como o pirarucu, quelônios e jacaré.

\section{A CRISE CIVILIZACIONAL NO ANTROPOCENO}

Leff (2009) discute estarmos vivendo uma crise no pensamento, na forma como a humanidade se relaciona com o conhecimento, de tal forma, uma crise civilizacional. Ela se apresenta nas formas de poluição, a falta de alimento e água potável, crise energética, os conflitos sociais, étnicos, disputas e exploração excessiva dos bens comuns e alterações nos padrões climáticos. Para Chakrabarty (2009; 2015), o atual cenário de crise é um somatório das ações humanas no ambiente, representada numa convergência entre as histórias do sistema terrestre, da vida e da civilização industrial.

O somatório da interação humano-ambiente é compreendido atualmente como a época do Antropoceno. O Antropoceno representa a caracterização humana como agente geológico (CRUTZEN, 2002; STEFFEN; CRUTZEN; MCNEILL, 2007; STEFFEN et al., 2011; STEFFEN et al., 2015). Para Steffen, Crutzen e McNeill (2007), isso fica evidenciado a partir de 1945, quando a "Grande Aceleração" intensifica as ações antrópicas no planeta, refletindo no aumento da população humana, no número de carros, atividades de turismo, uso da água e fertilizantes, entre outros (STEFFEN et al., 2011). De acordo com Steffen et al. (2011), as consequências dessas ações são observadas pelo aumento na concentração dos principais gases de efeito estufa $\left(\mathrm{CO}_{2}, \mathrm{~N}_{2} \mathrm{O}\right.$ e $\mathrm{CH}_{4}$ ) na atmosfera, áreas degradadas para a agricultura, e pelo aumento na taxa de extinção da biodiversidade global. As altas taxas de extinção das espécies podem ser entendidas com um genocídio não humano (DERRIDA, 2002).

Levantamentos da biodiversidade global estimam serem conhecidas aproximadamente 2 milhões de espécies animais, de um total de 5 a 11 milhões existentes (WWF, 2014; PEREIRA et al., 2010; DIRZO et al., 2014). No entanto estudos mostram que essa fauna sofre uma taxa extinção da ordem de 10 mil/ano (WWF, 2014) ou 0,01-1,0\% das espécies por década (COSTELLO, 2015). Ou seja, espécies são extintas sem sequer serem catalogadas. Para Costello (2015), essa quantificação atual assim como a previsão das futuras taxas de extinção tornam-se mais difíceis com o passar do tempo, devido ao fato de as causas mudarem ao longo do tempo, atrelado a um insuficiente monitoramento da biodiversidade.

Esse processo da perda de espécies das populações de animais silvestres é denominado de defaunação (GALETTI; DIRZO, 2013; DIRZO et al., 2014). Estudos mostram que esse processo já tenha ocorrido outras cinco vezes, e a atual seria a sexta grande extinção no planeta (KOLBERT, 2015; YOUNG et al., 2016; CEBALLOS; EHRLICH; DIRZO, 2017). A defaunação é resultado das 
ações dos humanos em escalas globais e locais, como mudanças climáticas, poluição oceânica, a caça direta, doenças endêmicas, espécies invasoras a supressão e modificação de habitats pelo desmatamento (DIRZO et al., 2014; YOUNG et al., 2016).

Estima-se que aproximadamente $50 \%$ do número de espécies animais já existentes na Terra foram extintas, devido à pressão da superpopulação humana, ao crescimento contínuo da população e excesso de consumo, especialmente pelos ricos (CEBALLOS; EHRLICH; DIRZO, 2017). As consequências da defaunação impactam as estruturas ecológicas e as funções/serviços ecossistêmicos, tais como os padrões evolutivos das espécies, a abundância, a ecologia dos biomas, a polinização, no controle biológico, alteração no ciclo dos nutrientes bem como na qualidade da água (DIRZO et al., 2014; YOUNG et al., 2016).

Para Ceballos, Ehrlich e Dirzo (2017), a humanidade está atrasada para planejar uma ação efetiva, pois o período é curto, provavelmente duas ou três décadas no máximo. Nesse sentido, um dos instrumentos para orientar a conservação da biodiversidade é a Lista Vermelha (Red List) da União Internacional para a Conservação da Natureza (IUCN). Trata-se de um levantamento periódico para identificar a situação da conservação de espécies e classificá-las em nove grupos a partir dos critérios: taxa de declínio da população, tamanho e distribuição da população, a área de distribuição geográfica e grau de fragmentação. As categorias de classificação de risco de extinção vão de Segura ou Pouco Preocupante (Least Concern, "LC") à Extinta (Extinct, "EX") (IUCN, 2014). Por vezes, listas paralelas sintetizadas por países apresentam dados conflitantes acerca do estado de extinção de determinadas espécie, nesses casos, algumas diretrizes são adicionadas, como por exemplo, se ela é endêmica ao território nacional ou não.

No Brasil, o Instituto Chico Mendes de Conservação da Biodiversidade (ICMBio) é o responsável por esses indicadores; vale ressaltar que a lista do ICMBio possui a categoria Regionalmente Extinta ou Extinta no Brasil (Regionally Extinct, "RE"), classificação essa que fica entre Extinta na Natureza (EW) e Criticamente em Perigo (CR) (ICMBIO, 2014). De acordo com a última lista divulgada nas portarias n. 444/2014 e n. 445/2014 do Ministério do Meio Ambiente, existem atualmente 1.173 espécies da fauna em extinção (BRASIL, 2014ab). São 299 invertebrados, 234 aves, 110 mamíferos, 80 répteis, 41 anfíbios, 353 peixes ósseos (310 de água doce e 43 marinhos), 55 peixes cartilaginosos (54 marinhos e 1 de água doce) e um peixe-bruxa que estão distribuídas em quatro categorias: 448 espécies vulneráveis (VU), 406 em perigo (EM), 318 em estado crítico (CR) e 1 extinto na natureza (EW).

Identificadas as espécies prioritárias, são criados os Planos de Ação Nacional para a Conservação das Espécies Ameaçadas de Extinção ou do Patrimônio Espeleológico (PAN). Os PANs identificam e orientam as ações prioritárias para combater as ameaças que põem em risco populações de espécies e os ambientes naturais e, assim, protegê-los (ICMBIO, 2014). Atualmente existem 55 planos de ação, divididos pelos grupos taxonômicos: aves, mamíferos, invertebrados terrestres, crustáceos, moluscos, peixes, répteis, anfíbios e flora.

\section{NECESSIDADES DO MANEJO E CONSERVAÇÃO DA FAUNA SILVESTRE}

As primeiras iniciativas de conservação enfatizavam a proteção de áreas florestais para manter a salvo o status cênico da natureza (FRANCO, 2013). Entendia-se que, ao retirar os moradores locais, a "natureza" retornaria ao estado de "intocabilidade" pela preservação. No presente trabalho, a conservação é entendida como a possibilidade de utilizar um recurso/bem natural de 
forma organizada respeitosa, consciente. Esta ocorre de forma in situ e ex situ, a primeira busca conservar seres vivos em seu local de origem, já a segunda condiz aos processos que envolvem a conservação fora do habitat natural. Nos dois casos, há potencialidades e dificuldades, por isso de acordo com Gastal e Saragoussi (2008, p.49), "a conservação ex situ quase sempre complementa as ações de conservação in situ", pois, enquanto a última tem um apelo estético e romântico, a segunda possibilita um acompanhamento e observação mais controlada.

Enfatizando a conservação in situ, destaca-se a estratégia de selecionar espécies animais para serem utilizadas como símbolos ou bandeiras (flagship species) (BARUA et al., 2011; SMITH et al., 2012). As espécies bandeiras possuem maior visibilidade num determinado ecossistema, mas não necessariamente é a espécie com maior contribuição/função ecológica. São espécies cativantes, pelo seu carisma ou apelo estético, com isso divulgam causa conservacionista. No caso do Brasil, conservar a fauna silvestre implica considerar, além dos fatores territoriais, os poucos recursos, a população humana sem acesso à alimentação, educação e saúde, elementos que dificultam as ações de conservação (MENEGUZZO; CHAICOUSKI, 2010).

Nesse sentido, o manejo pode contribuir na recuperação e conservação das unidades espaciais, estruturadas e complexas, cujos elementos, atores e fatores, sejam bióticos, físicos ou socioeconômicos, mantêm relação de interdependência (BERKES, 2009). De acordo com Mengak (2008), o manejo ou gestão da fauna ocorre há milhares de anos e tem como objetivos fornecer alimentos, vestimentas, proteção e abrigo aos humanos. Trata-se, portanto, de manipular ou gerir recursos para aumentar ou diminuir o tamanho da população de determinadas espécies em resposta aos objetivos da ação.

Na região amazônica, o manejo tem sido encarado como uma estratégia histórica para desenvolvimento local (MCGRATH et al., 2008). No entanto Berkes (2004; 2009) ressalta a necessidade de entendê-lo como uma ação compartilhada, envolvendo diversos agentes (indivíduos, comunidades, instituições ou órgãos) dividindo responsabilidades pela ação, sendo flexível, adaptativo e que promova uma aprendizagem social.

Se, por um lado, o manejo como prática adaptativa no Antropoceno pode ser entendido como domínio humano sobre as outras formas de vida, em especial pelas técnicas de domesticação, por outro, há quem perceba os outros animais não humanos como pessoas ou sujeitos-de-uma-vida (SINGER, 2010; REGAN, 2006) e, portanto, possuem direito a ter direito. Somam-se ao contexto das práticas de manejo na região amazônica, aspectos intersubjetivos da relação entre as sociedades ameríndias e os animais não humanos (VIVEIROS DE CASTRO, 1996; 2013; DESCOLA, 1998; 2012). No entanto, na atual conjuntura do panorama ambiental, onde há falta de alimentos e fontes de renda, a caça e pesca predatória tornaram-se as estratégias para a sobrevivência e constituição das sociedades humanas.

\section{A RELAÇÃO COM A FAUNA EM TEMPOS DE CRISE: casos na Amazônia}

Estudos mostram que a constituição da história humana se deu pela dependência da fauna e flora (ORLAND, 2004; THOMAS, 2010). Orland (2004) discute que a evolução das sociedades humanas pode ser entendida a partir da relação com os animais de acordo com cinco estágios, a saber: i) sociedades caçadoras/coletoras; ii) sociedades agrárias iniciais; iii) sociedades agrárias finais; iv) sociedades industriais iniciais; e v) sociedades industriais tardias.

Nesses estágios, o processo da domesticação da fauna é entendido como ponto de partida para a constituição da sociedade tal como é conhecida hoje, pois resulta em transformações 
biológicas para controlar a vida de determinada espécie animal ou vegetal (MAZOYER; ROUDART, 2010). Num sentido mais crítico da domesticação, autores refletem denotar aspectos de dominação, servidão ou mesmo a escravidão dos animais (SINGER, 2010; REGAN, 2006).

Na história recente da Amazônia, a relação entre humanos e não humanos foi marcada pela existência por mercados externos que há séculos fomentaram a predação contínua da fauna silvestre com objetivo de obter especificamente peles de animais (ANTUNES; SHEPARD; VENTICINQUE, 2014). Essa pressão ao longo de um século deixou vulnerável principalmente os seres da fauna aquática, fonte de vida dos ribeirinhos amazônicos (ANTUNES et al., 2016). Entenda-se ribeirinhos como membros de populações tradicionais que vivem em agrupamentos comunitários formados por várias famílias, localizados, ao longo dos rios e seus tributários, tais como lagos e paranás, construindo suas moradias nas várzeas e barrancos (LIRA; CHAVES, 2016; CASTRO; OLIVEIRA, 2016).

Essa forma particular de viver na Amazônia constitui-se pelo uso do território, manejo coletivo dos recursos locais, estabelecimento das relações sociais de trabalho, bem como, nas relações de compadrio e parentesco. Lira e Chaves (2016) citam que os ribeirinhos possuem uma relação particular com a natureza, traduzida num corpo de saberes técnicos, apropriação e conhecimentos sobre os ciclos naturais e os ecossistemas locais. Portanto conhecem/reconhecem as potencialidades que seus locais de vida possuem para fomentar sua permanência e sobrevivência, sejam de ambientes aquáticos ou terrestres, criando os meios de trabalho e estruturas culturais materiais e imateriais (CASTRO; OLIVEIRA, 2016).

Dessa forma, discutir o Antropoceno na Amazônia é antes de tudo, pensar como as formas de relação com o ambiente transformaram a relação entre ribeirinhos, os não ribeirinhos, pirarucus, jacarés e quelônios. Nas seções, são discutidos aspectos biológicos relacionados às três espécies e panoramas do manejo na Amazônia.

\subsection{Pirarucu, o bacalhau da Amazônia}

O pirarucu (Arapaima gigas) (Cuvier 1829) é considerado uma das maiores espécies entre os peixes de escama de água doce do mundo, chegando a medir 3 metros e pesar em torno de 200 kg (CASTELLO, 2008). De acordo com Castello, Stewart e Arantes (2013), estudos recentes mostram haver ao menos cinco espécies de pirarucus: A. gigas, A. mapae, A. arapaima, A. leptosoma e A. agassizii. No entanto os autores citam que, devido a pouca quantidade de estudos sobre a taxonomia da espécie, é provável que existam mais espécies de pirarucus.

Castello (2008) cita que o pirarucu habita principalmente áreas de planícies alagadas na bacia do Amazonas e Essequibo (Guiana Inglesa), incluindo florestas alagadas, rios, lagos, e algumas drenagens costeiras do Brasil. A distribuição geográfica do pirarucu geralmente é determinada por barreiras geográficas, como as quedas de água com correnteza forte impedindo sua passagem, preferindo habitar ambientes com correnteza fraca ou nula como os lagos. O pirarucu vive nos lagos de várzea, mas também pode ser encontrado nas praias dos rios e em alguns canais durante a época de seca, onde as espécies adultas formam o casal, que, com a subida do nível do rio, constrói o ninho na beira das florestas de restinga que circundam os ambientes de lago, ressaca e paraná (CASTELLO, 2008; CASTELLO; STEWART; ARANTES, 2013).

A pesca do pirarucu constituiu-se historicamente como uma importante fonte de alimento na Bacia Amazônica, e eles provavelmente desempenham papéis essenciais do ecossistema 
porque são grandes predadores (CASTELLO, 2008). A maior parte da pesca está concentrada na época da seca, já que, nesse período, os níveis baixos da água restringem a área disponível para os peixes, que ficam mais vulneráveis à pesca. Castello, Stewart e Arantes (2013) citam que a pesca é feita usando arpão, malhadeira, assim como anzóis e linhas; as malhadeiras têm sido cada vez mais usadas, enquanto o arpão representa um utensílio tradicional, feito desde os anos 1800, é preferido pelos pescadores experientes por ser mais seletivo. Além de ser uma iguaria tradicional da culinária amazônica, essa espécie já era produto de exportação desde o século XIX, quando eram exportadas da Amazônia em média 1200 toneladas do peixe (LOUREIRO, 2007).

Castello, Stewart e Arantes (2013) citam que a principal ameaça ao pirarucu é a sobrepesca, mas também a degradação dos habitats e a translocação de indivíduos para a aquicultura. E de acordo com os autores, as inciativas governamentais de estruturar o manejo da espécie como uma forma de combater a pesca predatória não foram efetivas. Isso porque na maioria dos países onde há iniciativas de manejo, assim como o Brasil, existem regras de tamanho mínimo de captura e defeso reprodutivo que são amplamente desrespeitadas pelos pescadores e, portanto, ineficazes em assegurar a sustentabilidade das populações de pirarucu (CASTELLO; STEWART; ARANTES, 2013).

A sobrepesca do pirarucu, ao longo de anos, fez com que na década de 80 fosse listada como espécie em extinção, com status de "vulnerável" na lista da IUCN, mas atualmente encontra-se fora dessa lista e do ICMBio (IUCN, 2014; ICMBIO, 2014). No Brasil, a regulamentação do manejo do pirarucu foi iniciada pelo IBAMA na sua Portaria 08/1996, e na Normativa 01/2005, mas, somente em 2015, o Estado do Amazonas regulamentou a pesca do Pirarucu (Arapaima spp) pelo Decreto 36.083/2015.

Dados do Instituto Brasileiro de Geografia e Estatística (IBGE, 2015) mostram que, em 2011, a produção de carne do pirarucu era de aproximadamente 1.000 toneladas; em 2013, a produção chegou a 2.301 toneladas; e em 2014, a produção alcançou 11.763 toneladas. Hoje é considerada a sétima carne mais consumida no país, a primeira é a tilápia (IBGE, 2015). Mesmo que haja a regulamentação para o manejo da espécie, Castello, Stewart e Arantes (2013) ressaltam que ainda não existe a tecnologia necessária para reproduzir o pirarucu em cativeiro, por isso a maioria das empresas de aquicultura dependem da coleta de indivíduos em ambiente natural.

No entanto não existem dados oficiais acerca da quantidade total de alevinos capturados, os locais de translocação, a sustentabilidade dessa captura e o impacto causado sobre as populações naturais (CASTELLO; STEWART, 2010). Dessa forma, se, sob a ótica do desenvolvimento e geração de renda, a pesca manejada alcança o seu objetivo, a falta de tecnologia e fiscalização podem afetar essa eficácia. O manejo, portanto, não trata apenas de sistematizar protocolos e procedimentos voltados ao manejo da espécie e beneficiamento da carne do pescado, mas também à sustentabilidade da população de pirarucus.

Apesar da escassez de informações acerca do pirarucu, Castello, Stewart e Arantes (2013) citam um modelo desenvolvido na Reserva de Desenvolvimento Sustentável Mamirauá (AM), localizada na região do médio Solimões. Esse modelo baseia-se nos conhecimentos de alguns pescadores experientes que utilizam uma metodologia para contar o número de pirarucus no momento que os indivíduos vêm à superfície para respirar. No modelo de manejo, todos os anos os pescadores realizam censos populacionais (contam pirarucu) em toda a área de manejo, em geral lagos. Na sequência, em colaboração com Instituto Mamirauá e o IBAMA, os pescadores utilizam informações para determinar cotas de pesca para o próximo ano. A supervisão dos 
procedimentos é realizada pelo IBAMA, autorizando ou não as cotas de pesca, ficando a cargo dos pescadores a responsabilidade por cumprir e fiscalizar as regras de manejo.

\subsection{Os quelônios da Amazônia}

De acordo com Van Dijk et al. (2014), são conhecidas 335 espécies de quelônios e, incluindo as subespécies, representam 453 táxons modernos, divididos em 14 famílias. No Brasil, podem ser encontradas 36 espécies. Somente na Amazônia brasileira são conhecidas 17 espécies continentais: sendo 15 aquáticas e duas terrestres. São 4 espécies da subordem pleurodira e 13 espécies cryptodira (VAN DIJK et al., 2014), a saber: Peltocephalus dumerilianus (cabeçudo) (Schweigger 1812), Podocnemis erythrocephala (irapuca ou calalumã) (Spix 1824), P. expansa (a tartaruga-da-amazônia, capitari ou viração) (Schweigger 1812), P. sextuberculata (o iaçá, pitiú ou cambéua) (Cornalia 1849) e o P. unifilis (tracajá ou zé-prego) (Troschel 1848). Destes a tartaruga-da-amazônia e o tracajá são as espécies mais consumidas em toda Amazônia (FERRARA et al., 2016).

Na Amazônia, historicamente, os quelônios foram utilizados para a fabricação de combustível, quando a gordura retirada dos ovos das espécies servia para a iluminação pública, e também na conservação de carnes, assim como na dieta dos ribeirinhos, na forma de mixira (SALERA JÚNIOR; BALESTRA; LUZ, 2016). Atualmente as espécies P. dumerilianus (Schweigger 1812), P. erythrocephala (Spix 1824), P. sextuberculata (Cornalia 1849) e $P$. unifilis encontram-se vulnerável (VU) na lista de extinção da IUCN, e somente a P. expansa (Schweigger 1812) é considerada de baixo risco de extinção nessa mesma lista. Essas mesmas espécies encontram-se fora da lista de animais em extinção do ICMBio (2014).

Tendo em vista a importância histórica da tartaruga-da-amazônia e do tracajá para as comunidades ribeirinhas da Amazônia no âmbito socioeconômico, Ferrara et al. (2016) citam que foram criadas portarias específicas do Instituto Brasileiro do Meio Ambiente e dos Recursos Naturais Renováveis (IBAMA), as quais normatizam sua criação em cativeiro (Portaria n. 142, de 30 de dezembro de 1992) e a comercialização de seus produtos e subprodutos (Portaria n. 70, de 23 de agosto de 1996). A Instrução Normativa (IN) do IBAMA n. 169, de 20 de fevereiro de 2008, institui e normatiza as categorias de uso e manejo da fauna silvestre em cativeiro no Brasil. Nessa IN, são encontradas a tartarugada-amazônia e o tracajá, e incluídas o iaçá e a muçuã como espécies que podem ser criadas, comercializadas e abatidas no Brasil.

Em 2015, o IBAMA (BRASIL, 2015), mediante a Instrução Normativa n. 07, instituiu e normatizou as categorias de uso e manejo da fauna silvestre em cativeiro, visando atender às finalidades socioculturais, de pesquisa científica, de conservação, de exposição, de manutenção, de criação, de reprodução, de comercialização, de abate e de beneficiamento de produtos e subprodutos. Nessa IN, foram elencadas as determinações para a criação o quelônios-de-água-doce, P. expansa, P. unifilis, P. sextuberculata e Kinosternon scorpidioides. No entanto, de acordo com a norma, os criadores comerciais apenas poderão ser instalados nas áreas de distribuição geográfica natural das respectivas espécies, sendo que os animais utilizados deverão apresentar potencial econômico para produção de matrizes e reprodutores (BRASIL, 2015).

No estado do Amazonas, no ano de 2017, a Secretaria de Estado de Meio Ambiente (SEMA, 2017) pela Resolução n. 26 do Conselho Estadual de Meio Ambiente do Estado do Amazonas (CEMAEAM), estabeleceu os procedimentos técnicos para criação e manejo comunitário de quelônios no Estado. De acordo com a resolução, são estabelecidos os procedimentos técnicos 
para a criação comunitária do P. expansa e P. unifilis, com fins comerciais pelas comunidades de populações tradicionais ribeirinhas no Estado do Amazonas. As comunidades interessadas devem comprovar, no mínimo, 5 anos de histórico de proteção dos ninhos e filhotes das referidas espécies, obedecendo às áreas prioritárias para essa ação, a saber: nível 1, área de proteção com grande população de quelônios ou populações ameaçadas; nível 2, área de conservação, uso e manejo dos quelônios pelos ribeirinhos; e nível 3, área de conservação, fora das áreas de proteção.

Além das deliberações legislativas, existem iniciativas como projetos de pesquisa, como o Projeto Pé-de-Pincha da Universidade Federal do Amazonas, os quais realizam o manejo e conservação de quelônios comunitária. Vidal e Costa (2007) descrevem que o manejo comunitário é composto por fiscalizações, identificação e coleta dos ovos, transferência dos ninhos das praias naturais para as artificiais, acompanhamento do nascimento e biometria dos filhotes e, por fim, a soltura da espécie na região coletada. Ressalta-se ainda que esse modelo de manejo se aplica para proteção de ninhos naturais, quando os ovos não são retirados do local e quando é realizado o transplante dos ninhos para outras áreas de proteção (ANDRADE et al., 2012). Vidal e Costa (2007) citam que a permanência dos filhotes nos berçários, por cerca de três meses após o nascimento, permite o aumento das chances de sobrevivência destes, quando são devolvidos ao ambiente natural.

Esses procedimentos de manejo sistematizados pelo projeto refletem a devolução de mais de 1.568.670 filhotes de quelônios no período de 1999 a 2012. Desses montantes, 43,6\% eram de tracajás (Podocnemis unifilis), 35,8\% de tartarugas-da-amazônia (P. expansa), 17,1\% de iaçás ou pitiús (P. sextuberculata) e 3,3\% de irapucas ou calalumãs (P. erytrocephala) (ANDRADE, 2012). Portanto as experiências construídas junto a esse projeto demonstram que técnicas e etapas do manejo de espécies, como o tracajá e a tartaruga-da-amazônia, em curso podem servir de orientação para comunidades ribeirinhas com potenciais para a criação comunitária desses dois quelônios.

\subsection{Alligators do Brasil}

Atualmente, são conhecidos oito gêneros com 25 espécies de crocodilianos distribuídos em todo o mundo, trópicos, subtrópicos, alcançando algumas regiões das zonas temperadas (PINCHEIRA-DONOSO et al., 2013). A família Alligatoridae contém quatro gêneros, Alligator, Caiman, Melanosuchus e Paleosuchus; no Brasil, são encontradas seis espécies, todas pertencentes à família Alligatoridae: Melanosuchus niger (jacaré-açú), Paleosuchus palpebrosus (jacarépaguá), Paleosuchus trigonatus (jacaré-coroa), Caiman crocodilus (jacaré-tinga), Caiman yacare (jacaré-do-pantanal) e Caiman latirostris (jacaré-do-papo amarelo) (RUEDA-ALMONACID et al., 2007). Na Amazônia, só não são encontradas as espécies de jacaré-de-papo-amarelo (Caiman latirostris) (Daudin 1802) e jacaré-do-pantanal (Caiman yacare).

As quatro espécies constam na Red List da IUCN como baixa preocupação (LC), e não constam da lista do ICMBio. Ao longo de quase duas décadas de criação racional de jacarés no Brasil, o objetivo principal era a obtenção de peles com melhor qualidade, ao contrário daquelas provenientes de animais capturados da natureza (SOUZA; SANTOS; CAMPOS, 2014).

Além do uso de peles, a criação de jacarés é realizada visando à produção de carne para o consumo local, e sua importância econômica estimula a presença do comércio ilegal. Somente a partir de 2003, no estado do Amazonas, têm sido criadas políticas públicas para utilizar o jacaré como recurso comum e fonte de renda legal (BOTERO-ARIAS; MARMONTEL; QUEIROZ, 2009). 
Em 2004, o governo do Estado do Amazonas iniciou a implementação do primeiro projeto de manejo experimental de jacarés na Amazônia brasileira, intitulado "Projeto Piloto para o Manejo de Jacarés na Reserva Mamirauá" (BOTERO-ARIAS; REGATIERI, 2013). A legalização do abate não só do jacaré, mas de outras espécies, é uma tendência para um mercado que busca transformar os animais silvestres em fontes renováveis de produtos de grande rentabilidade, contribuindo para a produção de alimentos e concorrendo, em custo de produção, com os animais domésticos (SOUZA; SANTOS; CAMPOS, 2014).

A mesma IN (BRASIL, 2015) que orienta de uso e manejo de quelônios, também determina as diretrizes para o manejo sustentado de crocodilianos das espécies, Caiman crocodilus, $C$. latirostris, C. yacare e Melanosuchus niger. De acordo com a instrução, o manejo em cativeiro com fins comerciais somente poderá ser instalado nas suas respectivas áreas de distribuição. A reprodução, nesse caso, é em cativeiro num sistema de ciclo fechado (Farming), ou sistema a partir da coleta de ovos ou filhotes na natureza e recria dos jovens em cativeiro (sistema tipo Ranching). Além dessas técnicas, a captura da espécie também ocorre extensivamente pela caça controlada de populações selvagens, conhecido por harvesting ou cropping (SCHú et al., 2015). Ressalta-se que as carnes de jacaré devem provir de criadouros comerciais autorizados pelo IBAMA e devem ser regulamentados por normas de qualidade do MAPA, da Agência Nacional de Vigilância Sanitária (ANVISA) e dos órgãos estaduais e municipais relacionados à qualidade de alimentos. Os jacarés, assim como os quelônios, além de serem fontes nutricionais, também são utilizados nas práticas da medicina tradicional local.

Eles são utilizados no preparo de chás ou pomadas para tratar desde problemas respiratórios a convulsões infantis, servindo também como substâncias cosméticas (SILVA, 2008). Mas, diferente das outras duas espécies, os jacarés são vistos com "maus olhos" pelos comunitários locais. Por ser um predador de topo, assim como o homem, há uma competição por espaços e alimentos no mesmo nicho, e isso pode provocar prejuízos mútuos (BARBOZA et al., 2013). Tratase, portanto, em determinados casos, de uma relação conflituosa, mas que possui um fundo de valor instrumental para os humanos.

\subsection{A relação entre o manejo das espécies}

De acordo com a legislação brasileira, a pesca, a caça e o abate, diretos de animais no ambiente, salvo as exceções amparadas pela lei, são considerados crime ambiental, e, portanto, passível de penalidades (BRASIL, 1998). No entanto, mesmo diante dessa situação, algumas espécies animais possuem liberação governamental de manejo, caça e pesca, tal como os pirarucus, jacarés e quelônios. Essa realidade é possível a partir da criação de acordo internacionais, como a Convenção sobre Comércio Internacional de Espécies da Fauna e Flora Silvestres Ameaçadas de Extinção (CITES, em inglês), órgão internacional criado na década de 1960, o qual funciona para garantir e controlar o comércio internacional de espécimes de animais selvagens e plantas desde que não ameace sua sobrevivência.

A CITES lista espécies silvestres em três apêndices: no I estão espécies ameaçadas de extinção, mas podem ser comercializadas em circunstâncias excepcionais; o II inclui espécies não necessariamente ameaçadas de extinção, mas seu comércio deve ser controlado para evitar a utilização incompatível com sua sobrevivência; o III são espécies protegidas em pelo menos um país, nesse caso a CITES controla o comércio externo. No caso dos jacarés, as quatro espé- 
cies amazônicas encontram-se listadas no apêndice II, com ressalvas para o Caiman crododilus (Linnaeus 1758) e Melanosuchus niger (Spix 1825), que se encontram também no apêndice I, mas que, no caso do Brasil, podem ser comercializadas. Todos os quelônios listados encontram-se no apêndice II. O pirarucu encontra-se no apêndice II da CITES.

Diante dos acordos internacionais e listas governamentais, pirarucus, jacarés e quelônios são semelhantes, e isso são elementos para iniciativas para desenvolver o manejo, e, como visto, a Amazônia é um locus para essas experiências. Para os ribeirinhos, a relações com esses animais representam fontes econômicas e nutritivas. O pirarucu e os quelônios representam maior fonte de alimento em comparação com o jacaré, pois este último é visto como "perigo" (BARBOZA et al., 2013). Dos quelônios, além da carne, também são aproveitados os ovos, e com a legislação em contínua regulamentação, o potencial de utilização aumenta (ANDRADE, 2012; SEMA, 2017). O pirarucu, assim como outros peixes, está presente na vida nos ribeirinhos, mas, da mesma forma que os quelônios, a pressão contínua e degradação do ambiente põem em risco a espécie (CASTELLO, 2008).

No âmbito geral, as três espécies sofreram e estão sofrendo pressão humana. Mas, de acordo com análises de Dirzo et al. (2014) e Young et al. (2016), por estarem em ecossistemas tropicais de água-doce, o risco aumenta, pois o número de animais em extinção é nove vezes maior nesses locais, quando comparado ao dos animais em ecossistemas marinhos.

\section{CONSIDERAÇÕES FINAIS}

Ao longo da história, as espécies em questão tiveram significativa importância ao modo de vida ribeirinha, entrecruzando seu cotidiano, sendo um de seus principais recursos alimentares, fontes medicinais e artesanato. Hoje, há uma recaracterização, que, por um lado aproxima, quando não ribeirinhos se beneficiam ou passam a conhecer as espécies, mas também distancia humanos e não humanos, ao passo de serem "objetificadas", tal como ocorreu com o gado, o frango, o suíno e demais animais de criação/fazenda. Mesmo essas transformações estando em curso, cada vez mais a máquina antropocêntrica precisará de outros subsídios para funcionar, a questão é saber quem serão os outros a serem fagocitados pelo Antropoceno.

O manejo como estratégia de conservação de espécies ameaçadas de extinção, quando bem realizadas podem beneficiar qualquer população humana. Mas, para que isso ocorra, é necessário enfatizar a relação sensível entre humano e não humano e aglutinar os conhecimentos ecológicos, biológicos e etológicos para dar maior eficiência ao manejo. Portanto, assim como no passado, o manejo, agora legalizado, regulamentado e normatizado, seja para conservar ou domesticar as chamadas espécies silvestres, pode ser considerado uma solução para mitigar o processo de extinção e enfrentar a crise ambiental. Essa é nova forma de nos relacionar com a fauna silvestre em tempos de crise ambiental.

\section{REFERÊNCIAS}

AMAZONAS (Estado). Decreto n. 36.083, de 23 de julho de 2015. Disponível em: <http://www.normasbrasil. com.br/norma/decreto-36083-2015-am_287404.html>. Acesso em: 30 maio 2018.

ANDRADE, Paulo César Machado. O projeto Pé-de-Pincha em números: a conservação comunitária de tracajás (Podocnemis unifilis). In: ANDRADE, Paulo César Machado (Org.). Manejo comunitário de quelônios no Médio Amazonas e Juruá - Projeto Pé-de-Pincha. Manaus: Gráfica Moderna, 2012. p. 15-88. 
ANDRADE, Paulo César Machado et al. Sistematização dos métodos utilizados pelo projeto Pé-de-Pincha para a conservação comunitária de quelônios - transferência de ninhos e berçários. In: ANDRADE, Paulo César Machado (Org.). Manejo comunitário de quelônios no Médio Amazonas e Juruá - Projeto Pé-dePincha. Manaus: Gráfica Moderna, 2012. p. 89-156.

ANTUNES, André Pinassi et al. Empty forest or empty rivers? A century of commercial hunting in Amazonia. Science Advances, v. 2, n. 10, p. 1-14, out. 2016.

ANTUNES, André P.; SHEPARD JR., Glenn H.; VENTICINQUE, Eduardo M. O comércio internacional de peles silvestres na Amazônia brasileira no século XX. Boletim do Museu Paraense Emílio Goeldi. Ciências Humanas, Belém, PA, v. 9, n. 2, p. 487-518, maio/ago. 2014.

BARBOZA, Rafael Sá Leitão et al. Plano de manejo comunitário de jacarés na várzea do baixo rio Amazonas, Santarém - PA, Brasil. Biotemas, Florianópolis, SC, v. 26, n. 2, p. 215-26, jun. 2013.

BARUA, Maan et al. Defining flagship uses is critical for flagship selection: a critique of the IUCN climate change flagship fleet. Ambio, v. 40, n. 4, p. 431-5, jun. 2011.

BERKES, Fikret. Evolution of co-management: role of knowledge generation, bridging organizations and social learning. Journal of Environmental Management, v. 90, n. 5, p. 1692-702, abr. 2009.

Rethinking community-based conservation. Conservation Biology, v. 18, n. 3, p. 621-30, 2004.

BOTERO-ARIAS, Robinson; MARMONTEL, Miriam; QUEIROZ, Helder Lima de. Projeto de manejo experimental de jacarés no Estado do Amazonas: abate de jacarés no setor Jarauá - Reserva de Desenvolvimento Sustentável Mamirauá, dezembro de 2008. UAKARI, v. 5, n. 2, p. 49-58, dez. 2009.

BOTERO-ARIAS, Robinson; REGATIERI, Sandro A. Construindo as bases para um Sistema de Manejo Participativo dos Jacarés Amazônicos. Tefé, AM: IDSM, 2013. 68p.

BRASIL. Ministério do Meio Ambiente. Lista Nacional Oficial de Espécies da Fauna Ameaçadas de Extinção. Portaria MMA n. 444, de 17 de dezembro de 2014a. Disponível em: <http://www.icmbio.gov.br/cepsul/ images/stories/legislacao/Portaria/2014/p_mma_444_2014_lista_esp\%C3\%A9cies_ame\%C3\%A7adas_ extin\%C3\%A7\%C3\%A3o.pdf>.

. Ministério do Meio Ambiente. Lista Nacional Oficial de Espécies da Fauna Ameaçadas de Extinção - Peixes e Invertebrados Aquáticos. Portaria MMA n. 445, de 17 de dezembro de 2014b.

. Instrução Normativa IBAMA n. 07/2015, de 30 de abril de 2015. Institui e normatiza as categorias de uso e manejo da fauna silvestre em cativeiro, e define, no âmbito do Ibama, os procedimentos autorizativos para as categorias estabelecidas. Disponível em: <http://www.ibama.gov.br/phocadownload/ fauna/faunasilvestre/2015_ibama_in_07_2015_autorizacao_uso_fauna_empreendimentos.pdf>.

. Lei n. 9.605, de 12 de fevereiro de 1998. Dispõe sobre as sanções penais e administrativas derivadas de condutas e atividades lesivas ao meio ambiente, e dá outras providências. Disponível em: <http://www.planalto.gov.br/ccivil_03/leis/L9605.htm>.

CASTELLO, Leandro. Lateral migration of Arapaima gigas in floodplains on the Amazon. Ecology of Freshwater Fish, v. 17, n. 1, p. 38-46, 2008.

CASTELLO, Leandro; STEWART, Donald. J. Assessing CITES non-detriment findings procedures for arapaima in Brazil. Journal of Applied Ichthyology, v. 26, n. 1, p. 49-56, fev. 2010.

CASTELLO, Leandro; STEWART, Donald J.; ARANTES, Caroline C. O que sabemos e precisamos fazer a respeito da conservação do pirarucu (Arapaima spp.) na Amazônia. In: FIGUEIREDO, Ellen Sílvia Amaral (Org.). Biologia, conservação e manejo participativo de pirarucus na Pan-Amazônia. Tefé, AM: IDSM, 2013. p. 17-32.

CASTRO, Roberta Rowsy Amorim de; OLIVEIRA, Myriam Cyntia Cesar de. Os termos "populações" e "comunidades" tradicionais e a apropriação dos conceitos no contexto amazônico. Mundo Amazónico, Colômbia, v. 7, n. 1-2, p. 47-70, 2016. 
CEBALLOS, Gerardo; EHRLICH, Paul R.; DIRZO, Rodolfo. Biological annihilation via the ongoing sixth mass extinction signaled by vertebrate population losses and declines. PNAS, Ecology, v. 114, n. 30, p. E6089-E6096, jul. 2017.

CONVENÇÃO SOBRE COMÉRCIO INTERNACIONAL DE ESPÉCIES DA FAUNA E FLORA SILVESTRES AMEAÇADAS DE EXTINÇÃO (CITES). What is CITES? 2017. Disponível em: <https://www.cites.org/eng/disc/what.php>. Acesso em: 10 jun. 2017.

php>.

Appendices I, II and III. Abr. 2017. Disponível em: <https://www.cites.org/eng/app/appendices.

CHAKRABARTY, Dipesh. The anthropocene and the convergence of histories. In: HAMILTON, Clive; BONNEUIL, Christophe; GEMENNE, Bonneuil. The anthropocene and the global environmental crisis. Rethinking modernity in a new epoch. Oxfor, RN/New York, NY: Routledge Environmental Humanities, 2015. p.44-56.

The climate of history: four theses. Critical Inquiry, v. 35, n. 2, p. 197-222, 2009.

COSTELLO, Mark J. Biodiversity: the known, unknown, and rates of extinction. Current Biology, v. 25, n. 9, R362-R383, maio 2015.

CRUTZEN, Paul J. Geology of mankind: the Anthropocene. Nature, v. 415, n. 23, p. 23, jan. 2002.

DERRIDA, Jacques. O animal que logo sou. São Paulo: Editora UNESP, 2002.

DESCOLA, Philippe. Más al lá de naturaleza y cultura. 1. ed. Buenos Aires: Amorrortu, 2012.

Estrutura ou sentimento: a relação com o animal na Amazônia. Mana, Rio de Janeiro, v. 4, n. 1, p. 23-45, abr. 1998.

DIRZO, Rodolfo et al. Defaunation in Antropoceno. Science, v. 345, n. 6195, p. 401-6, jul. 2014.

FERRARA, Camila Rudge et al. História natural e biologia dos quelônios amazônicos. In: BALESTRA, Rafael Antônio Machado (Org.). Manejo conservacionista e monitoramento populacional de quelônios amazônicos. Instituto Brasileiro do Meio Ambiente e dos Recursos Naturais Renováveis. Brasília: Ibama, 2016. p. 15-28.

FRANCO, José Luiz de Andrade. O conceito de biodiversidade e a história da biologia da conservação: da preservação da wilderness à conservação da biodiversidade. História, São Paulo, v. 32, n. 2, p. 21-48, jul./dez. 2013.

GALETTI, Mauro; DIRZO, Rodolfo. Ecological and evolutionary consequences of living in a defaunated world. Biological Conservation, v. 163, número especial, p. 1-6, jul. 2013.

GASTAL, Maria Luiza; SARAGOUSSI, Muriel. Os instrumentos para a conservação da biodiversidade. In: BENSUSAN, Nurit (Org.). Seria melhor mandar ladrilhar? Biodiversidade: como, para que e por quê? Brasília: Editora UnB, 2008.

INSTITUTO BRASILEIRO DO MEIO AMBIENTE E DOS RECURSOS NATURAIS RENOVÁVEIS (IBAMA). Instrução Normativa n. 169, de 20 de fevereiro de 2008. Instituir e normatizar as categorias de uso e manejo da fauna silvestre em cativeiro em território brasileiro, Disponível em: <http://www.mma.gov.br/port/conama/ legiabre.cfm?codlegi=585>. Acesso em: 30 maio 2018.

. Instrução Normativa n. 001, de 1o de junho de 2005. Proibir anualmente a pesca, o transporte, a armazenagem e a comercialização do pirarucu (Arapaima gigas) no estado do Amazonas, durante o período de 1 을 de junho de a 30 de novembro. Disponível em: <http://www.ibama.gov.br/sophia/cnia/ legislacao/IBAMA/IN0001-010605.PDF>. Acesso em: 30 maio 2018.

Portaria IBAMA n. 08, de 2 de fevereiro de 1996. Estabelece normas gerais para o exercício da pesca na bacia hidrográfica do Rio Amazonas. Disponível em: <http://www.icmbio.gov.br/cepsul/images/ stories/legislacao/Portaria/1996/p_ibama_08_1996_regulamentapescabaciahidroraficarioamazonas. pdf>. Acesso em: 30 maio 2018. 
. Portaria n. 70, de 23 de agosto de 1996. Normatiza a comercialização de produtos e subprodutos das espécies de quelônios. Disponível em: <http://www.ibama.gov.br/sophia/cnia/legislacao/PRT7096. htm>. Acesso em: 30 maio 2018.

Portaria n. 142, de 30 de dezembro de 1992. Controla as transações comerciais das espécies: Tartaruga da Amazônia (Podocnemis expansa) e Tracajá (Podocnemis unifilis). Disponível em: <http://www. ibama.gov.br/sophia/cnia/legislacao/IBAMA/PT0142-301292.PDF>. Acesso em: 30 maio 2018.

INSTITUTO BRASILEIRO DE GEOGRAFIA E ESTATISITICA (IBGE). Produção da Pecuária Municipal - 2015, Rio de Janeiro, v. 43, p. 1-49, 2015.

INSTITUTO CHICO MENDES DE CONSERVAÇÃO DA BIODIVERSIDADE (ICMBio). Ministério do Meio Ambiente. Roteiro Metodológico para Avaliação do Estado de Conservação das Espécies da Fauna Brasileira. Ago. 2014. Disponível em: <http://www.icmbio.gov.br/portal/images/stories/biodiversidade/fauna-brasileira/ avaliacao-do-risco/Roteiro_Metodologico_Avaliacao_Fauna_Brasileira_2014.pdf>.

INTERNATIONAL UNION FOR CONSERVATION OF NATURE (IUCN). IUCN Red List, 2014. Disponível em: $<$ http://www.iucnredlist.org/about/introduction>.

KOLBERT, Elizabeth. A sexta extinção: uma história não natural. Tradução de Mauro Pinheiro. 1. ed. Rio de Janeiro: Intrínseca, 2015.

KRITSCH, Rebeca. The Amazon's flora and fauna. AMAZON Initiative, WWF, fev. 2009.

LEFF, Enrique. Complexidade, racionalidade ambiental e diálogo de saberes. Educação e Realidade, Porto Alegre, RS, v. 4, n. 3, p. 17-24, set./dez. 2009.

LIRA, Talita de Melo; CHAVES, Maria do Perpétuo Socorro Rodrigues. Comunidades ribeirinhas na Amazônia: organização sociocultural e política. Interações, Campo Grande, MS, v. 17, n. 1, p. 66-76, jan./mar. 2016.

LOUREIRO, Antônio José Souto. O Amazonas na época imperial. 2. ed. Manaus: Valer, 2007. 328p.

MAZOYER, Marcel; ROUDART, Laurence. História das agriculturas no mundo: do neolítico a crise contemporânea. São Paulo: Editora UNESP, 2010.

MCGRATH, David G. et al. Constructing a policy and institutional framework for na ecosystem-based approach to managing the lower Amazon floodplain. Environment, Development and Sustainability, $\mathrm{n}$. 10, p. 677-95, 2008.

MENEGUZZO, Isonel Sandino; CHAICOUSKI, Adeline. Reflexões acerca dos conceitos de degradação ambiental, impacto ambiental e conservação da natureza. Geografia, Londrina, PR, v. 19, n. 1, p. 181-5, 2010.

MENGAK, Michael T. Wildlife management. Forests and Forestry in the Americas: an encyclopedia. Society of American Foresters, International Society of Tropical Foresters, fev. 2008. Disponível em: http://www. encyclopediaofforestry.org/index.php/Wildlife_Management

ORLAND, Mary A. Roots of the modern environmental dilemma: a brief history of the relationship between humans and wildlife. Marine BioConservation Society, 2004. Disponível em: <http://marinebio.org/oceans/ conservation/moyle/ch1-3/>.

PEREIRA, Henrique M. et al. Scenarios for Global Biodiversity in the 21st Century. Science, v. 330, n. 6010, p. 1496-501, dez. 2010.

PINCHEIRA-DONOSO, Daniel; BAUER, Aaron M.; MEIRI, Shai; UETZ, Peter. Global taxonomic diversity of living reptiles. Plos One, v. 8, n. 3, p. 1-10, 2013.

REGAN, Tom. Jaulas vazias: encarando o desafio dos direitos animais. Porto Alegre: Lugano, 2006.

RUEDA-ALMONACID, Jose Vicente et al. Las tortugas y los cocodrilianos de los países andinos del trópico. Bogotá, Colombia: Conservación Internacional, 2007. 538p.

SALERA JÚNIOR, Giovanni; BALESTRA, Rafael Antônio Machado; LUZ, Vera Lúcia Ferreira. Breve histórico da conservação dos quelônios amazônicos no Brasil. In: BALESTRA, Rafael Antônio Machado (Org.). Manejo 
conservacionista e monitoramento populacional de quelônios amazônicos. Instituto Brasileiro do Meio Ambiente e dos Recursos Naturais Renováveis. Brasília: Ibama, 2016. p. 11-4.

SCHÚ, Camila et al. Manejo reprodutivo de crocodilianos. Revista Investigação Medicina Veterinária, Franca, SP, v. 14, n. 1, p. 104-9, 2015.

SECRETÁRIA DE ESTADO DE MEIO AMBIENTE (SEMA). Conselho Estadual de Meio Ambiente do Estado do Amazonas. Resolução n. 26, de agosto de 2017. Estabelece os procedimentos técnicos para a criação e o manejo comunitário de quelônios no Estado do Amazonas. Diário Oficial do Estado do Amazonas. Disponível em: <https://sogi8.sogi.com.br/Arquivo/Modulo113.MRID109/Registro1283218/resolucao\%20 cemam\%20n\%2026\%20de\%2018082017.pdf>.

SILVA, Andréa Leme da. Animais medicinais: conhecimento e uso entre as populações ribeirinhas do rio Negro, Amazonas, Brasil. Boletim do Museu Paraense Emílio Goeldi. Ciências Humanas, Belém, PA, v. 3, n. 3, p. 343-57, set./dez. 2008.

SINGER, Paul. Libertação animal. São Paulo: WMF/Martins Fontes, 2010.

$\mathrm{SMITH}$, Robert J. et al. Identifying Cinderella species: uncovering mammals with conservation flagship appeal. Conservation Letters, v. 5, n. 3, p. 205-12, 2012.

SOUZA, Bruna Crislane da Silva; SANTOS, Gracielle Alves dos; CAMPOS, Rogério Manoel Lemes de. Carne de jacaré - Revisão de Literatura. Revista Eletrônica Nutritime, v. 11, n. 6, p. 3741-54, nov./dez. 2014.

STEFFEN, Will; CRUTZEN, Paul J.; MCNEILL, John R. The Anthropocene: are humans now overwhelming the great forces of nature. AMBIO: A Journal of the Humans Environment, v. 36, n. 8, p. 614-21, 2007.

STEFFEN, Will et al. The trajectory of the Anthropocene: the great acceleration. The Anthropocene Review, v. 2, n. 1, p. 81-98, 2015.

STEFFEN, Will et al. The Anthropocene: conceptual and historical perspectives. Philosophical Transactions of the Royal Society, A, n. 369, p. 842-67, jan. 2011.

THOMAS, Keith. O homem e o mundo natural: mudanças de atitudes em relação as plantas e aos animais (1500-1800). São Paulo: Companhia das Letras, 2010.

VAN DIJK, Peter Paul et al. Turtle Taxonomy Working Group. Turtles of the World. 7. ed. Annotated Checklist of Taxonomy, Synonymy, Distribution with maps, and Conservation Status. Chelonian Research Monographs, v. 7, n. 5, p. 329-479, 2014.

VIDAL, Marcelo Derzi; COSTA, Tiago Viana da. Manejo comunitário de quelônios: a parceria ProVárzea/ Ibama - Pé-de-Pincha. In: MACHADO, Paulo Cesar (Org.). Criação e manejo de quelônios no Amazonas. Manaus: Ibama, ProVárzea, 2007.

VIVEIROS DE CASTRO, Eduardo. A inconstância da alma selvagem e outros ensaios de antropologia. São Paulo: Cosac \& Naify, 2013.

. Os pronomes cosmológicos e o perspectivismo ameríndio. Mana, Rio de Janeiro, v. 2, n. 2, p. 115-44, out. 1996.

WORLD WIDE FUND FOR NATURE (WWF). How many species are we losing? 2014. Disponível em: <http:// wwf.panda.org/about_our_earth/biodiversity/biodiversity/>.

YOUNG, Hillary S. et al. Patterns, causes, and consequences of Anthropocene defaunation. Annual Review of Ecology, Evolution, and Systematics, v. 47, p. 333-58, nov. 2016. 


\section{Sobre os autores:}

Wagner de Deus Mateus: Doutor em Ciências do Ambiente e Sustentabilidade na Amazônia pela Universidade Federal do Amazonas e Mestre em Educação em Ciências na Amazônia, pela Universidade do Estado do Amazonas. E-mail: wagnermthus@gmail.com

Mariana Baldoino: Doutoranda do Programa de Pós-Graduação em Ciências do Ambiente e Sustentabilidade na Amazônia e Mestre em Sociedade e Cultura na Amazônia pela Universidade Federal do Amazonas. E-mail: mariana_baldoino@yahoo.com.br

Elisa Ferrari Justulin Zacarias: Mestranda no Programa de Pós-graduação em Ciências do Ambiente e Sustentabilidade na Amazônia da Universidade Federal do Amazonas e graduada em Ciências Sociais pela Universidade Estadual Paulista Júlio de Mesquita Filho, Araraquara, em Licenciatura Filosofia, pelas Faculdades Integradas Claretianas e em Serviço Social pela Universidade Paulista. E-mail: elisa.justulin@gmail.com

Maria Inês Gasparetto Higuchi: Doutora em Antropologia Social pela Brunel University of London. Mestre em Ecologia Humana pela Michigan State University. Graduada em Psicologia pela Pontifícia Universidade Católica do Paraná. Pesquisadora titular do Instituto Nacional de Pesquisas da Amazônia, coordenando o Laboratório de Psicologia e Educação Ambiental. E-mail: higuchi.mig@gmail.com 
